\title{
Research on Diagnostic Accuracy and Related Factors of CT for Intra-Thoracic Anastomotic Leak
}

\author{
Caiyun Liu \\ Department of Radiology, the Third Affiliated Hospital of Xuzhou \\ Medical University, Xuzhou, Jiangsu, 221000, China
}

\begin{abstract}
Objective: To evaluate the accuracy of $\mathrm{CT}$ in the diagnosis of esophageal fistula (Anastomotic leakage). Methods: Retrospective analysis of 186 cases of suspected postoperative esophageal postoperative intracavitary AL patients, 70 cases take oral $60 \%$ compound diatrizoate (contrast agent) CT examination and 116 cases take oral contrast agent CT examination. The CT images of oral or non-oral contrast agents were observed by two imaging physicians (Physician 1 and Physician 2, respectively). Kappa test was used to evaluate the consistency of two physicians in diagnosing AL. The variables related to AL were screened by Fisher exact test, Pearson $\chi^{2}$ test and univariate analysis. Multivariate logistic regression analysis was used to confirm the reliable signs of AL. Results AL49 cases, no AL137 cases. The diagnostic sensitivity and specificity of AL were $79.6 \%, 83.7 \%, 83.9 \%$ and $84.7 \%$ respectively. The positive predictive value was significantly improved by oral contrast (from $40.6 \%$ to $89.7 \%$, from $39.4 \%$ to $96.5 \%, \mathrm{P}<0.05$ ). The sensitivity and specificity were $83.3 \%, 86.7 \%, 97.5 \%$ and $97.5 \%$ respectively. There was also a high correlation between the accumulation of gas and anastomotic effusion. The univariate analysis showed that all of them were related to $\mathrm{AL}(\mathrm{P}<0.05)$. Multivariate analysis showed independent correlation of contrast agent spillover. Conclusion CT can be a comprehensive assessment of postoperative esophageal AL-related signs, CT combined with oral contrast agent can improve the diagnostic accuracy and the contrast agent spillover is the most reliable signs.
\end{abstract}

Keywords: anastomotic leak, tomography; contrast agent spillover 


\section{Introduction}

Postoperative esophageal anastomotic leakage (AL) is one of the most serious complications after esophagectomy and gastrointestinal reconstruction. The morbidity and mortality are $1.3 \%-21 \%, 10 \%-35 \%$ [1-2 ]; Divided into the neck $\mathrm{AL}$ and thoracic $\mathrm{AL}$, the thoracic AL diagnosis, treatment is not timely, easy to quickly develop mediastinal inflammation, a higher mortality rate. Early diagnosis is the key to reducing postoperative complications and mortality. The traditional view that the upper gastrointestinal imaging diagnosis of esophageal postoperative thoracic AL is the preferred method of examination [3-4], can show the contrast agent in the residual esophagus, anastomosis, generation organ cavity dynamic movement, can determine the fistula Location, but also to estimate the size of the fistula, but the sensitivity and positive predictive value of low (positive predictive value, PPV), and can not observe the esophageal, mediastinal, pulmonary lesions. With the development of CT, CT is the main method to diagnose AL in thoracic esophagus. It can not only observe the integrity of anastomosis, but also clearly observe the infection of tissues, organs, mediastinum, thoracic cavity and lung tissue around the anastomosis. Therefore, $\mathrm{CT}$ and thin-layer reconstruction, CPR and multi-window technique combined with oral contrast agent were used to observe the CT signs of thoracic AL after esophagectomy to improve the diagnostic accuracy and confirm the reliable signs of AL.

\section{Materials and methods}

\subsection{General Information}

Retrospective analysis of the Third Affiliated Hospital of Xuzhou Medical University in December 2010 to September 2016 meets the following criteria patients. Inclusion criteria: (1) postoperative esophageal cancer; (2) clinical suspicion of intrathoracic AL due to high fever, chest pain or dyspnea; (3) CT scan and clinical data were complete and follow-up time $\geqslant 3$ months. Exclusion criteria: (1) no esophageal fever, chest pain, dyspnea; (2) imaging and clinical data is not complete; (3) follow-up time $<3$ months. The final 186 patients were included in this study, 35 females, male 151 cases, aged $45-82$ years, median age 67 years. Including 49 cases of AL (confirmed by endoscopy), 11 females, 38 males, aged 45-80 years, median age 65 years; 38 cases of esophageal cancer, 11 cases of gastric cardia; 37 cases were placed Recovered esophageal covered stent was cured, 12 patients were placed into the stomach, feeding tube and antiinflammatory treatment support and cure. No AL 137 cases (at least 3 months follow-up confirmed). Clinical manifestations: persistent high fever, severe chest pain, difficulty breathing. The median time from the first operation to the CT examination was 7 days, ranging from 2 days to 28 days. 


\subsection{Inspection methods}

Siemens Emotion multislice spiral CT was used to scan the chest and upper abdomen continuously from the upper edge of the clavicle to the renal hilum plane. 70 cases of supine examination bed were treated with $60 \%$ compound diatrizoate (China, Hunan Hansen Pharmaceutical Company) 20ml-100ml after CT examination, 116 cases of oral contrast agent, scanning position: arms on the move, supine position, the first advanced, layer thickness, layer spacing are $5 \mathrm{~mm}$. (WW: 400HU-500HU, WL: $-100 \mathrm{HU}$ ), bone window (WW: 1000HU, WL: $700 \mathrm{HU}$ ), mediastinal window (WW: 350HU-400HU, WL: 40HU) : 1500HU, WL: $500 \mathrm{HU})$ reconstruction. The reconstructed increment was $0.7 \mathrm{~mm}$ after the thinlayer reconstruction of the 4 kinds of window technique, and then transferred to the CT workstation through the PACS system. Then the sagittal plane and the coronal plane were reconstructed. Reformation, CPR).

\subsection{Image analysis}

(1) There was a spillover of contrast agent around the anastomosis, and the diagnosis of AL was confirmed. (2) The diagnosis of AL was based on the diagnostic criteria anastomosis around the product gas or the presence of effusion, product gas, suggesting AL. 2 = indeterminate; $3=$ possibly AL; $4=$ positive for AL. The diagnosis of AL was given a 5-point confidence scale: $0=$ definitely not $\mathrm{AL} ; 1$ = probably not AL; Scores of 1, 2 for the diagnosis of negative, 3,4 for the diagnosis of positive. (1) free gas: including the mediastinal product gas or pneumothorax or mediastinal pneumothorax, pneumothorax exist, free contrast agent spillover; (2) (3) effusion: including mediastinal effusion or pleural effusion or mediastinum, pleural effusion exist at the maximum level of the longest diameter $\geqslant 10 \mathrm{~mm}$ to determine the presence of effusion, $<10 \mathrm{~mm}$ determine the absence of effusion, The location of effusion and anastomotic position; (4) associated with the anastomotic effusion Pneumatic gas: the maximum level of the largest diameter $\geqslant 10 \mathrm{~mm}$ or $\geqslant 3$ bubbles and anastomosis to determine, $<10 \mathrm{~mm}$ or $<3$ bubbles and anastomosis Mouth; (5) anastomotic defect. Determine the spillover contrast agent, free gas, effusion and anastomotic position relationship: distance anastomosis $<15 \mathrm{~mm}$ to determine the anastomosis around, $\geqslant 15 \mathrm{~mm}$ away from the anastomotic. The number of CT signs associated with AL was recorded by endoscopy or follow-up results as the gold standard.

\subsection{Statistical methods}

SPSS20.0 software package was used for statistical analysis. Kappa test was used to evaluate the consistency of two physicians in diagnosing AL. To evaluate the sensitivity, specificity, PPV, negative predictive value (NPV) and accuracy of CT and single sign in the diagnosis of AL by endoscopy or follow-up results. The number of cases was used to describe the count data. Contrast agent spill test was performed by Fisher exact test. Pearson $\chi 2$ test was used to test the accumulation 
of gas, effusion and product gas around the anastomotic stoma. On the basis of univariate analysis, variables with statistical significance were screened out, and multivariate Logistic regression was used for analysis. $\mathrm{P}<0.05$ considered the difference was statistically significant.

\section{Results}

\subsection{The results of comprehensive $\mathrm{CT}$ signs to diagnose $\mathrm{AL}$}

AL49 cases (Figure 1-3), no AL137 cases. Two physicians using a comprehensive CT signs of the consistency of AL is better, total CT, oral contrast agent $\mathrm{CT}$, without oral contrast agent $\mathrm{CT}$ diagnosis of $\mathrm{AL} \mathrm{K}$ values were $0.691,0.899,0.588, \mathrm{P}$ values were $<0.05$ (Table 1,2 ). The diagnostic sensitivity of the physician 1 and the physician 2 were $79.6 \%$ and $83.7 \%$, respectively, and the specificity was $83.9 \%$ and $84.7 \%$, respectively. Oral contrast agents significantly increased PPV (from $40.6 \%$ to $89.7 \%, \mathrm{P}<0.05$; from $39.4 \%$ to $96.5 \%, \mathrm{P}<0.05$ ).

\subsection{CT signs of the results of the diagnosis of $\mathrm{AL}$}

Two doctors using a single CT signs of diagnosis of AL better consistency, contrast agent spill, around the anastomotic effusion, anastomotic gas, and anastomotic-related effusion of gas, effusion, gas diagnosis AL $\mathrm{K}$ value 0.057 , $0.519,0.537,0.697, \mathrm{P}$ values $<0.05$ (Table 3 ). The sensitivity and specificity of the two agents were $83.3 \%, 86.7 \%$ and $97.5 \%$, respectively. The univariate analysis showed significant correlation with $\mathrm{AL}(\mathrm{P}<0.05))$. The univariate analysis showed that there was a positive correlation with $\mathrm{AL}(\mathrm{P}<0.05) 4)$. In 12 cases, 13 cases were anastomotic, 24 cases were local anastomosis, 25 cases were anastomotic stoma, 25 cases were anastomotic, 24 cases were anastomotic. Multivariate analysis showed that contrast agent spillage was the most reliable predictor of AL (Table 5).

\section{Discussion}

Esophageal postoperative AL is a serious and refractory complication. Thoracic $\mathrm{AL}$ is associated with a lower incidence of $\mathrm{AL}$ in the neck, with $6.5 \%$ morbidity and $10 \%$ to $15 \%$ mortality. Delayed diagnosis (ie, onset of clinical symptoms after $24 \mathrm{~h}$ clearly diagnosed patients) cause a higher mortality described early diagnosis (24h) AL and esophageal mortality was no significant correlation, delay in the diagnosis of AL patients with oral saliva, gastric acid, gastric juice and gastric contents in the pleural suction, more vulnerable to fistula Into the mediastinum, mediastinal infection caused mediastinal abscess, if the infection perforation mediastinal pleura, then cause pleural effusion, empyema, rapid progress of this pathological process caused by the body reaction (body temperature, pulse speed, the final toxic shock, Organ failure), mortality 
increased up to $18 \%$. Therefore, early diagnosis of $\mathrm{AL}$ is the key to reducing mortality.

\subsection{The diagnosis value of $\mathrm{CT}$ to $\mathrm{AL}$}

The sensitivity, specificity $(79.6 \%, 83.7 \%, 83.9,84.7 \%)$ of CT diagnosis were higher than those of Doeksen A and so on, and the sensitivity, specificity $(65 \%$, $77 \%$ Due to differences in sample content and research methods. In this study, $\mathrm{CT}$ thin layer recombination combined with $\mathrm{CPR}$ and multiple window technique were used to observe and analyze the CT signs of thoracic AL after suspected esophageal surgery, especially the lack of typical symptoms and signs. The clinicians were highly suspicious of AL. Using the above method, Such as the mouth, a small amount of gas in the esophagus into the fistula around the anastomosis, mediastinal or pleural cavity, the formation of free small bubbles, digestive juice, a small amount of gas infiltration secondary inflammatory reaction caused by encapsulated fluid accumulation of gas, for early, timely diagnosis of AL To provide help to improve the diagnostic sensitivity and specificity; and the literature using 10mm thickness of suspicious colonoscopy after AL CT signs. Lan Guobin, etc. [5] reported that the appropriate choice of reconstructed images and observation window in the best way to display images, to improve the detection rate of lesions is essential.

The results of this study show that the accuracy of CT diagnosis of AL was $82.8 \%, 84.4 \%$, and reported in the literature [2] CT can be diagnosed $65 \%-89 \%$ of patients with AL is similar. Strauss C and so on 97 patients with esophageal postoperative thoracic AL patients were prospectively studied, respectively, on the $3 \mathrm{~d}, 7 \mathrm{~d}$ after thoracic CT scan and 7th line of water-soluble contrast agent in the upper gastrointestinal imaging contrast agent spill and CT display Anastomotic around the product gas, mediastinal product gas or pneumothorax as the diagnostic criteria, the results show that CT has a higher sensitivity and NPV.

\subsection{The CT signs of chest AL after esophageal}

This group of CT signs of the diagnosis of AL: contrast agent spill, around the anastomotic effusion, anastomotic gas around the anastomotic-related effusion gas, effusion, product gas, anastomotic defect, and the literature [2] (Anastomotic wall thickening, anastomotic defect, effusion, fluid product gas and liquid gas level, pleural effusion) are not consistent; this study using oral contrast agent to improve CT diagnostic efficacy. Kim TH et al [2] described the suspicious line of non-oral contrast agent CT examination, found that anastomotic wall defect and anastomotic site of a large number of product fluid accumulation of gas is an independent risk factor for the prediction of AL. Most of the false-negative cases in this group were non-oral contrast-agent $\mathrm{CT}$ examination; therefore, suspicious AL patients in the CT examination in the course of oral administration of contrast agents may delay the best timing of surgery, increased mortality. Power $\mathrm{N}$ and other reports around the anastomotic effusion is an independent predictor of AL risk factors, the study includes the colon and small intestine AL, the control group of patients with partial hepatectomy. Gervaz Pand other descriptions to 
exclude CT images show contrast agent spill in patients with anastomotic-related extra-cavity free gas, effusion and right colon AL was significantly related; white blood cell count $>9 \times 109$, anastomotic site extracorporeal gas, Effusion $>500 \mathrm{~mL}$ for the prediction of AL independent risk factors.

The results show that the contrast agent spillover is the most accurate CT diagnosis of AL signs. This and Huiberts AA et al that the contrast agent spillover is an independent risk factor for predicting $\mathrm{AL}$, can improve the accuracy of CT diagnosis, the best image shows the contrast near the anastomosis to improve diagnostic accuracy is essential to the results consistent . But with Khoury $\mathrm{W}$ and other descriptions of contrast agent spillover diagnosis of AL sensitivity of only $17 \%$ and Lynn ET and other descriptions of $32 \%$ of the results inconsistent, taking into account the sample composition and research methods, reported in different parts of the AL and different diagnosis method. $94 \%$ of the surgeons believe that the contrast agent spill and AL-related, and according to the spillover contrast dose and clinical manifestations to choose appropriate treatment. Kaur P and other studies of a single CT signs and AL correlation, found that anastomotic peritoneal gas is sensitive and specific signs of contrast agent spillover is a reliable sign; but the study includes only 28 cases of suspected postoperative colon AL patients, And CT diagnostic criteria fuzzy.

This study has some limitations: (1) This study is retrospective study, there may be selection bias. (2) not all of the suspicious patients with oral contrast agent CT examination, may lead to bias results. (3) observation of AL-related CT signs are subjective, the results may be biased.

CT can be a comprehensive assessment of esophageal postoperative thoracic AL-related signs, CT combined with oral contrast agent can improve the diagnostic accuracy, contrast agent spillover is the most reliable signs of diagnosis of AL.

\section{References}

[1]Low DE. Diagnosis and management of anastomotic leaks after esophagectomy. Gastrointest Surg, 2011, 15(8):1319-1322.

[2] Kim TH, Kim JH, Shin CI, et al. CT findings suggesting anastomotic leakand predicting the recovery period following gastric surgery. Eur Radiol, 2015, 25(7):1958-1966.

[3]Jones CM, Clarke B, Heah R, et al. Should routine assessment of anastom-otic integrity be undertaken using radiological contrast swallow after oesophagectomy with intra-thoracic anastomosis? Best evidence topic (BET). Int J Surg, 2015, 20:158-162.

[4] Honing J, Pultrum BB, van der Jagt EJ, et al. Routine or on demand radiological contrast examination in the diagnosis of anastomotic leakage after esophagectomy. J Surg Oncol, 2009, 100(8):699-702.

[5]Et al.Comparison of the diagnostic value of standard lung window, ventral window and fat window in gastrointestinal perforation pneumoperitoneum and its role in soft reading. Journal of Clinical Radiology, 